\title{
Performance comparison of multicast MANET CRN system's based on POS scheme with different types of router protocol algorithms
}

\author{
Basma Nazar Nadhim, Sarab Kamal Mahmood \\ Electrical Engineering Department, College of engineering, Mustansiriyah University, Iraq
}

\begin{tabular}{l}
\hline Article Info \\
\hline Article history: \\
Received May 30, 2019 \\
Revised Jun 1, 2019 \\
Accepted Jun 15, 2019 \\
\hline
\end{tabular}

\section{Keywords:}

CRN

MANET

MST

SMT

SPT

\begin{abstract}
A multi-layer multi-hop mechanism, with one of three different types of router protocol algorithms (Steiner minimal tree (SMT), shortest path tree (SPT) and minimal spanning tree (MST)) was employed for the construction of multi-cast MANET CRNs. The probability of success (POS) used as the channel assignment scheme, is dependent on the availability of the channel, and the required transmission time. It was applied to the network after the conversion of the network's random topology into one of the three algorithms (SMT, SPT or MST). This facilitated the selection of an efficient channel for the transmission of the CR user's data, under the effect of the Rayleigh fading channel. Three idle probability circumstances (i.e., $P_{I}=0.1$, $0.5,0.9)$, and different network parameters, were used to compare the performance of the CRN with three routing protocols in terms of throughput, and packet delivery rate (PDR). According to the simulation results, for a high traffic load of PUs $\left(P_{I}=0.1\right)$ at different network parameters, the CRN with the SPT algorithm performed better than the CRN with the SMT or MST algorithm.
\end{abstract}

Copyright $\odot 2020$ Institute of Advanced Engineering and Science. All rights reserved.

Corresponding Author:

Basma Nazar Nadhim,

Electrical Engineering Department,

Mustansiriyah University, Baghdad, Iraq.

Email: besma.nazar@yahoo.com

\section{INTRODUCTION}

In the last decade, wireless services have gained rapid popularity. Numerous countries have adopted the fixed spectrum allocation methodology, in which a majority of the available radio spectrum has already been assigned for different services [1-3]. However, a major chunk of the assigned spectrum has been employed sporadically and the range of geographical variations in terms of the usage of assigned spectrum falls between $15 \%$ and $85 \%$ accompanied by a high variance in time [4]. In fact, as per a recent study done by the Federal Communications Commission (FCC), majority of such licensed spectrums were still unoccupied for large time periods [5, 6]. We have put forward dynamic spectrum access (DSA), also referred to as cognitive radio, as a substitute policy to allow efficient use of the radio spectrum [7,8]. Thus, it can be considered as a potential technique that can be applied in future wireless communications to address the spectrum scarcity issue [9]. As per the FCC, cognitive radios (CRs) are radio systems that can allow conducting spectrum sensing uninterruptedly, dynamically detect spectrums that are unused and then functions in those spectrum holes in which there are idle licensed (primary) radio systems [10]. In other words, in terms of CRN, secondary users (SUs) could employ spectrum access opportunities to perform unlicensed transmissions when the licensed spectrum is not occupied by primary users (PUs) [11-13]. In Next Generation (xG) networks, employing cognitive radios can help identify unutilised spectrum as well as spectrum sharing with no disadvantaged interjecting with other users (Spectrum sensing), capturing the best available spectrum that is in par with the communication demands of the user (Spectrum management), 
maintain tractable communication exigencies when shifting to better spectrum (Spectrum mobility) and offer an equitably spectrum that allow assigning method amongst cohabitation $\mathrm{xG}$ users (Spectrum sharing ) $[4,14,15]$. Based on this definition, there are two key characteristics pertaining to the cognitive radio: Cognitive capability that allows information sensing for the radio technology from its radio environment, and reconfigurability that allows dynamic programming of the radio based on the radio environment [4, 14]. Figure 1 presents the needed mission pertaining to adaptive operation for open spectrum, which is also referred as the cognitive cycle. The key steps in the cognitive cycle include spectrum analysis, spectrum sensing and spectrum decision [14]. Numerous key applications are associated with cognitive radio. For instance, for radio interoperability, cognitive radio plays a key technology in the US military (i.e. JTRS program), future mobile base stations and public safety (i.e. SAFECOM program). For multi-hop ad hoc networks, CR is regarded as the radio platform. Multicast is a key service that the ad hoc networks need to support [16]. A mobile ad-hoc network (MANET) includes mobile nodes with no requirement for infrastructure [17]. MANET can be considered as a self-organising network without infrastructure, wherein each of the participating devices can receive as well as send data along with mobility model that is independent. The MANET ad hoc network has the ability to change locations as well as configure itself [18]. Multicasting is employed when there is a need for applications to send the same data multiple destinations. The communication costs for applications can be decreased with multicasting, when there is a need to send the same data to multiple recipients. In place of sending through multiple unicast, multicasting helps to reduce the link bandwidth consumption, delivery delay and router processing [19]. Classification of the existing multicast routing protocols pertaining to the MANETs could be done into mesh-based and treebased. The difference between these protocols lies based on the path redundancy between receivers and senders. Tree-based protocols offer just a single path between receivers and senders, while mesh-based protocols offer multiple paths [20, 21]. In recent years, there have been numerous routing protocols pertaining to CRNs that have been put forward and examined. More commonly, these protocols focus on either picking the channel that possesses the maximum average spectrum-availability time or the best-quality channel. In a CRN, both the needed transmission time and spectrum-availability time can considerably affect routing and network connectivity. Specifically, a significant reduction in CRN performance spectrumavailability time could result when there is smaller average spectrum-availability time of an assigned channel than what is needed for the transmission time over that channel. Even worse, this issue becomes even more vital in multi-hop CRNs when there are a lot of multiple links involved. Network performance can be enhanced by employing a diverse channel quality as well as spectrum availability more efficiently, provided these are considered by the cognitive routing protocol design [22]. Apart from employing efficient channel assignment to select the best channel for data transmission in multi hop multicast cognitive radio network, the manner in which network topology's connection also plays an important role in enhancing the system performance. The function of tree-based multicasting protocols is based on the tree construction for the overall graph that connects all the multicast groups in an acyclic subgraph together. In a tree structure, through a single path, every node could reach out to any of the other nodes. Two fundamentally separate approaches can be considered for the construction of multicasting trees: employing minimum cost trees (MCTs) or 'shortest path trees' (SPTs). The former approach is aimed at decreasing the overall edge cost of the tree, while the latter approach helps to reduce each receiver's distance from the sender [23].

In this research paper, we have employed the multi-layer multi hop multicast MANET CRN that included three types of router protocol algorithms (shortest path tree (SPT), Steiner minimal tree (SMT) and minimal spanning tree (MST)) for CRN. To enhance the network performance with regards to the throughput and packet delivery rate (PDR), we employed the probability of success (POS) as the channel assignment scheme, which is applied to the network post transformation of the network's random topology to one of the three router protocol algorithms. This allows selecting an efficient channel for data transmission based on the channel's availability and the needed transmission time. A comparison of the multi-layer multi-hop multicast MANET CRN system's performance along with these three algorithms as well as POS scheme was done to check if each of the router protocol algorithms shows the best performance versus others at various traffic loads of PU and different network parameters.

The rest of the paper is structured as follows: Section 2 presents the related work of practices and methods for multicasting network channel assignment and the routing protocol for undirected graph. Section 3 presents the router protocol algorithms for a multicasting network. The system model for the recommended multicast protocol is presented in Section 4. Lastly, the simulation outcomes and conclusions are presented in Sections 5 and 6, respectively. 


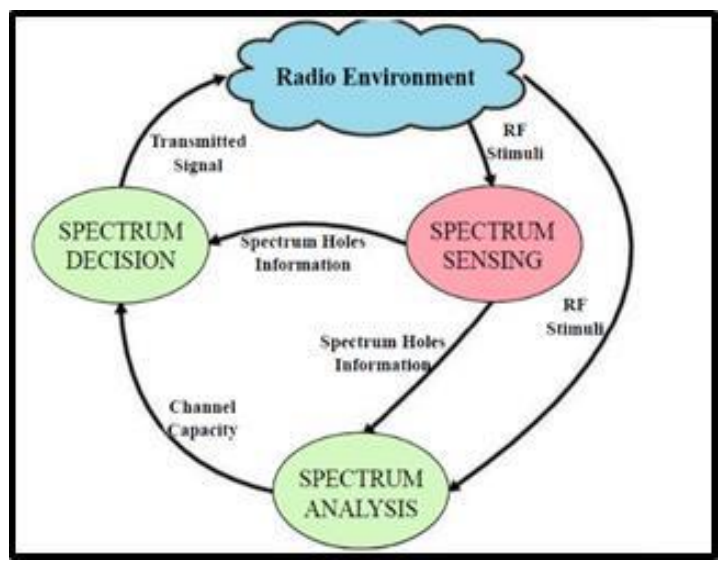

Figure1. Cognitive radio cycle

\section{RELATED WORKS}

In cognitive radio networks, multicasting seems a challenging issue because of the dynamic nature pertaining to the available spectrum opportunities for secondary users. The multicast cognitive radio network's performance is improved with regards to the throughput and various parameters by employing different methods and techniques for channel assignment as well as constructing the routing tree from random undirected topology [23]. To establish efficiency, there is a need for tree-shaped topologies pertaining to multicast connections. Transmission of a minimum number of data packets could be done in parallel to several destinations along the tree branches, where duplication is performed only for tree branches [24]. In [24], identification of Steiner trees pertaining to the topology of multicast connections for communication networks can be done when the primary aim is cost optimisation. In [25], presentation of the method pertaining to transforming undirected topology to Steiner tree was done. In [26], a novel routing protocol was put forward for multichannel CRNs, which uses a probabilistic metric. This approach relies on probabilistically determining the available capacity pertaining to each channel over every CR-to-CR link, while also considering primary radio (PR). In [27], CoCast was put forward as an ad hoc multicast protocol pertaining to cognitive radio-based MANETs. CoCast mitigates the scalability issue of ODMRP with regards to the number of multicast sources that use multiple cognitive radio channels. For building the minimumenergy multicast tree, a low complexity approximation algorithm guarantees bounded performance, which converts the multicast issue to a directed Steiner tree issue as mentioned in [28]. In terms of the cognitive radio networks, this approximation algorithm considers the energy that was utilised for sensing the spectrum opportunities, while its constructed multicast trees were found to be adaptive towards the traffic load pertaining to the primary network. In [29], a cross-layer optimisation approach has been put forward for the multicast video in CR networks. The modelling of the CR video multicast is done as an optimisation problem by accounting for important design factors such as video rate control, scalable video coding, spectrum sensing, modulation, scheduling, dynamic spectrum access, primary user protection and retransmission. For a multiuser single-transceiver cognitive radio network (CRN), the coordinated spectrum access issue has been regarded in [30]. Our goal here is to increase the sum-rate that has been realised through all contending cognitive radio users with regards to both transmission rate and spectrum assignment. In cognitive radio mesh networks, the multicast routing as well as channel allocation problem was accounted in [31]. An algorithm was put forward that concierges switching latency and channel heterogeneity. The algorithm focuses on decreasing the end-to-end delay, and simultaneously minimising the degradation of throughput by employing a dynamic programming approach. In [22], a novel routing metric was put forward for multi-hop CRN that falls under Rayleigh fading channel. This routing metric tries to maximise the success probability pertaining to a given CR transmission by accounting for both required transmission times as well as average residual spectrum-availability. With regards to this metric, a routing protocol was built for multi-hop CRN, namely MaxPoS. In [32], for the throughput maximisation in cognitive radio networks, overlapping and nonoverlapping channel assignment algorithms were accounted.

In [33], a routing algorithm has been put forward that makes use of the expected transmission count metric (ETX) pertaining to multi hop wireless cognitive network to choose high-quality channels to perform routing on a hop-by-hop basis. In [34], a cross-layer multicasting routing protocol has been put forward to stream video over cognitive radio networks to improve the received video's overall quality in the multicast group. In [35] and [36], MST and SPT are employed as routing protocols pertaining to multilayer multicast multi hop CRN along with POS scheme employed as channel assignment. The network's performance is 
improved with regards to throughput and PDR versus employing other schemes. As routing algorithms, EXT with SPT and MST are used for multicast multi hop while the POS scheme was employed as channel assignment to select an efficient channel for data transmission based on the channel availability and the needed time for transmission as presented in [37].

04

\section{ROUTER PROTOCOL ALGORITHMS FOR WIRELESS MULTICASTING}

The analysis and design of wireless multicast employs different algorithms and techniques. In this section, three types of router protocol algorithms (minimal spanning tree (MST), shortest path tree (SPT) and Steiner minimal tree (SMT)) have been introduced as mentioned below:

\subsection{Shortest Path Tree (SPT) Algorithm}

The SPT is a kind of tree created from an undirected graph. An undirected graph has weights that are non-negative and a node specially designated as root or source. The shortest path tree is the path having minimum total weight starting from the source and going towards each of the destinations in the graph. In case there is a change in the source node, the SPT should be re-created, making it more complex. The shortest path tree can be created with Dijkstra's algorithm having an overall running time which is equal to $(m+n \log n)$, where $m$ represents the amount of links (edges) that join the nodes while $n$ denotes the number of vertices. Figure 3 shows two SPTs having node (a) as source node for the weighted graph shows in Figure 2. The total weight of the edges in both the SPTs cannot be same. In Figure 3a it is 18, whereas it is 17 in Figure 3b. The purpose is to obtain the group of edges joining all nodes where the sum of the weights of the edges is minimum from the root to every node $[38,39]$.

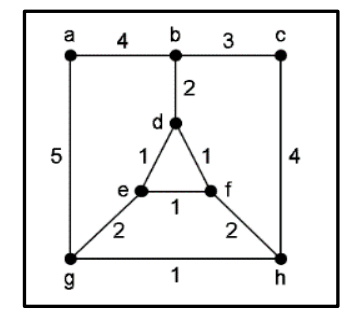

Figure 2. A weighted graph

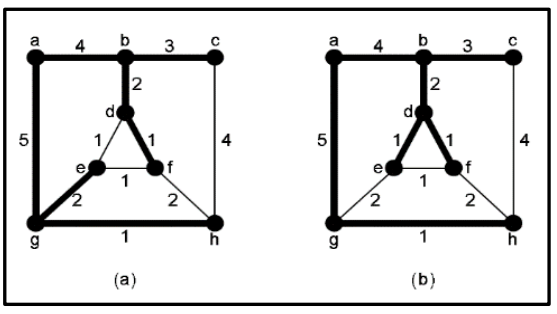

Figure 3. A shortest-paths tree rooted at vertex a

\subsection{Minimal Spanning Tree (MST) Algorithm}

The minimal spanning tree can be obtained by covering all the nodes in the undirected graph. It obtains a path having minimum sum of all the edges' total weights for every destination, and does not contain any loop. Many distinct MSTs are possible, for a single undirected graph having same total weight. Moreover, there is no need of a starting node (that is, the source). MST is flexible where any node can be selected as a source. Thus, if there is a change in the source node, then the tree need not be re-created. A minimal spanning tree can be created using Kruskal's technique which has a total running time equivalent to $(\mathrm{m} \log \mathrm{m})$. Figure 4 shows the MST for the graph in Figure 1. The total weight of the edges in this MST is 14. It is noteworthy that in case of MST, the root (source) node always requires more hops to cover all the nodes in comparison to SPT for the same graph. In Figure 4, the source node requires 4 hops to get to the last vertex in the MST, whereas in SPT, the source node requires just 3 hops to get to the last node as given in the Figure $3[35,38,39]$.

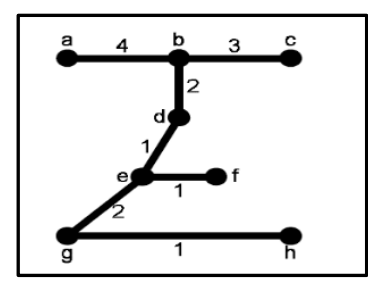

Figure 4. A minimal spanning tree 


\subsection{Steiner Minimal Tree (SMT) Algorithm}

A Steiner minimal tree spans a specific subset of nodes as against a spanning tree where all the nodes are spanned. For SMT, the nodes are distributed in two sets: nonterminal and terminal nodes. The terminal nodes are those nodes which are to be included in the SMT solution. The SMT cost is equal to the total weight of the edges. A Steiner tree possibly has certain nonterminal nodes to minimise the cost. Let $\mathrm{V}$ represent the set of nodes. As a rule, there is a terminal set $\mathrm{L} \subset \mathrm{V}$ and a metric identifying any 2 vertices' distance from each other in V. The purpose is to obtain a connected subgraph that spans every one of the terminals having minimum total cost. A renowned technique to obtain an SMT is by using an MST (minimal spanning tree). First, we build the metric closure on L, i.e. an entire graph with vertices $\mathrm{L}$ and edge weights equalling the shortest path lengths. Then, we determine an MST on the closure, wherein every edge matches one shortest path on the actual graph. Lastly, the MST is transmuted back to a Steiner tree by supplanting every edge with the shortest path and certain direct post processing for eliminating any likely cycle. Figure 5a depicts an undirected graph $G$ with terminal vertices $L=\{v 1, v 2$, v3, v4, v5 $\}$ and nonterminal vertices $\{\mathrm{u} 1, \mathrm{u} 2, \mathrm{u} 3, \mathrm{u} 4\}$. From Figure $5 \mathrm{c}$ and Figure $5 \mathrm{~d}$, it can be said that prior to adding $\{\mathrm{u} 1, \mathrm{u} 2\}$, the tree's total weight is 22 , whereas post adding $\{\mathrm{u} 1, \mathrm{u} 2\}$, it is reduced to 17 . This means the Steiner points (nonterminal vertices) play a role in decreasing the total cost of the tree [38, 39].

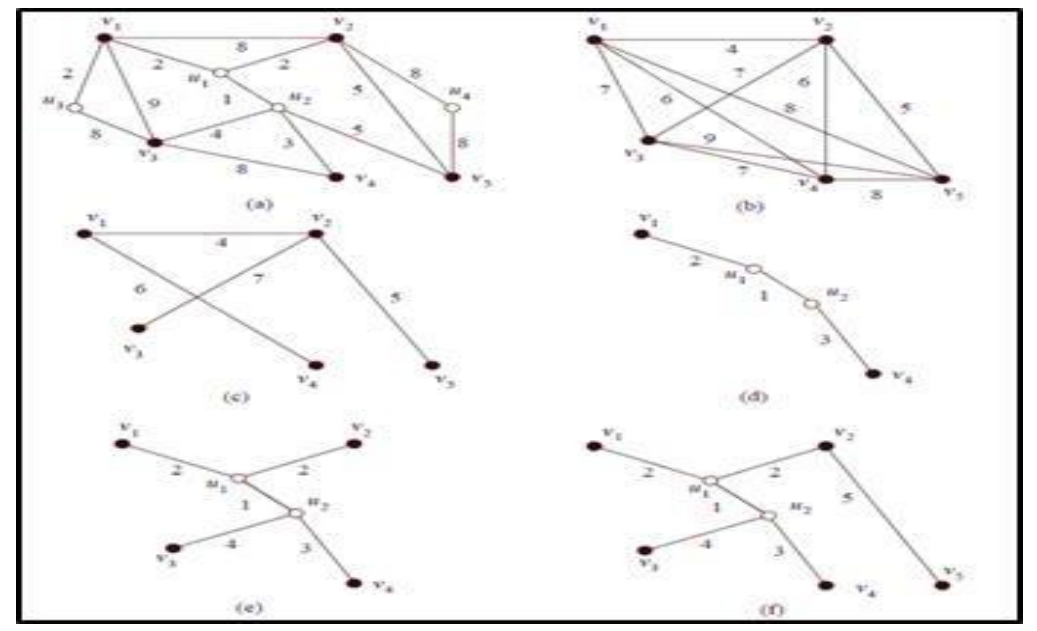

Figure 5. A Steiner minimal tree

\section{SYSTEM MODEL FOR MULTICAST MANET CRN}

This paper study considers multilayer multicast multi-hop MANET CRN with three kinds of router protocol algorithms (Steiner minimal tree (SMT), shortest path tree (SPT) and minimal spanning tree (MST)) for video transmission from the source to destination nodes over a solo session. First, an undirected graph with set of $\mathrm{N}$ vertices is produced within a square area. The algorithms outlined in section (3) are deployed for constructing MANET CRN. Several PU channels (M) are obtainable between the source and every destination node. The status model of every primary user (PU) channel is the Markov model, which switches between two states (idle and busy). A busy state indicates that the channel cannot be utilised by SU, while an idle state means that the channel is not utilised by PU. For all channels, the bandwidth (BW) set is the same. The POS scheme is deployed on the network as channel assignment for improving the network performance. For organising the CRN transmissions, a common control channel (CCC) is presented [35, 37]. The close form term for probability of success $\left(P O S_{j}^{(i-k)}\right)$ between any two nodes $\mathrm{i}$ and $\mathrm{j}$ in multilayer MANET CRN over channel $\mathrm{j}$ which contains the available channel (C) of CRN is expressed in [22] as in (1):

$$
\operatorname{POS}_{j}^{(i-k)}=\exp { }^{\left(\frac{-T_{(j)}^{(i-k)}}{\mu_{j}}\right)}
$$

where $\mu_{j}$ is the average availability time of spectrum in (in sec) for channel $\mathrm{j}$ and $T_{r_{j}}^{(i-k)}$ is the requisite transmission time in (in sec/packet) for sending a packet from node $\mathrm{i}$ to $\mathrm{k}$ over channel $\mathrm{j}$ where it can be stated as in (2) [22]: 


$$
T_{r_{j}}^{(i-k)}=\frac{D}{R_{j}^{(i-k)}}
$$

where D is the packet size (in bits) and $R_{j}^{(i-k)}$ is the data rate (in bit/sec) between nodes $\mathrm{i}$ and k over channel $\mathrm{j}$ which can be stated as in (3) [22]:

$$
R_{j}^{(i-k)}=(B W) \log _{2}\left(1+\frac{P_{r_{(j)}^{(i-k)}}^{\left(i N_{0}\right.}}{B W *}\right.
$$

where $N_{0}$ signifies the thermal power density in (Watt/Hz), $B W$ is the channel bandwidth and $P_{r(j)}^{(i-k)}$ signifies the received power from transmitter i to receiver $\mathrm{j}$ which can be stated as in (4) [22]:

$$
P_{r_{j}}^{(i-k)}=\frac{p_{t}}{d^{n}}\left(\frac{\lambda}{4 \pi}\right)^{2}\left(\xi_{(j)}^{(i-k)}\right)
$$

where $p_{t}$ is CR's transmission power, $\mathrm{d}$ is the distance between any two nodes, $\mathrm{n}$ is the path loss exponent, and $\xi_{(j)}^{(i-k)}$ is the channel power gain between nodes $\mathrm{i}$ and $\mathrm{k}$ over channel j. For Rayleigh fading, $\xi_{(j)}^{(i-k)}$ is exponentially distributed with mean $1[22]$.

\section{COMPUTER SIMULATION AND RESULTS}

Computer simulations for multicast MANET CRN with multi-layer multi hop were performed. Three different routing protocol algorithms (SMT, SPT and MST), together with a probability of success (POS) channel assignment scheme, were used to assess the network performance in terms of throughput and packet delivery rate (PDR), under the effect of the Rayleigh fading channel. Three values for idle probability (i.e. $P_{I}=0.1,0.5$ and 0.9 ) and the various network parameters used for comparison purposes, are displayed in Table 1. MATLAB R2018a was employed to carry out the simulations.

Table 1. System Parameters

\begin{tabular}{cc}
\hline Parameter & Value/Type \\
\hline Network area & $200 \mathrm{~m}^{*} 200 \mathrm{~m}$ \\
No. of Nodes & 30 \\
No. of Terminal nodes $(\mathrm{Nt})$ & 20 \\
No. of Nonterminal nodes (Nnt) & 10 \\
Topology tree & (SMT, SPT and MST) \\
No. of CR source & One source \\
No of primary channel $(\mathrm{M})$ & 15 \\
PU channel model & Markov model \\
Idle probability $\mathrm{P}_{\mathrm{I}}$ & {$[0.10 .50 .9]$} \\
Average availability time $\left(\mu_{j}\right)$ & Range from $2 \mathrm{~ms}$ to $45 \mathrm{~ms}$ \\
Bandwidth (BW) & $1 \mathrm{MHz}$ \\
Packet size (D) & $4 \mathrm{~KB}$ \\
Transmission power $(\mathrm{Pt})$ & $0.1 \mathrm{Watt}$ \\
Channel used & Rayleigh fading channel \\
Path loss exponent $(\mathrm{n})$ & $10^{-8} \mathrm{~W} / \mathrm{Hz}$ \\
Thermal noise power $\left(N_{0}\right)$ &
\end{tabular}

\subsection{Performance Evaluation of Multicast MANET CRN Under The Impact of Channel Bandwidth}

The throughput and PDR performance of multilayer multi hop multicast MANET CRN, with regards to the channel bandwidth with three different types of tree algorithms (SMT, SPT and MST), and three idle probability values $\left[\mathrm{P}_{\mathrm{I}}=0.1,0.5\right.$, and 0.9], are presented in Figures 6 and 7 respectively. POS was used as the channel assignment scheme in all the CNRs. According to the results attained, the data rate is proportional to the bandwidth of the channel. This indicates that an increase in the channel's bandwidth, improved the performance of the CRN tree algorithms. It was also observed that in terms of throughput and PDR, an increase in the $\mathrm{P}_{\mathrm{I}}$ value enhanced the performance of the CRN at the three algorithm protocols. This is because a high $P_{I}$ value raises the probability, that suitable channels will be available for transmission by CR users at a low traffic load of PU. At high and moderate idle probability values $\left[\mathrm{P}_{\mathrm{I}}=0.9\right.$, and 0.5$]$, it was observed from Figure 6 that the throughput performance of the CNR using the SMT routing tree 
algorithm, is comparable to the performance of the CRN with the MST algorithm at $15.5 \%$ and $15.49 \%$, while outperforming the CRN with the SPT algorithm by $24 \%$ and $26.3 \%$ at $\mathrm{P}_{\mathrm{I}}=0.9$ and 0.5 respectively. At busy traffic of the channel by primary users, where $\mathrm{P}_{\mathrm{I}}=0.1$, the CRN with the SPT algorithm outperformed the CRN with SMT and MST algorithms by $46 \%$ and $88.72 \%$ respectively. As for $\mathrm{P}_{\mathrm{I}}=$ 0.9 and 0.5 , the PDR performance of the CNR using the SMT tree, is comparable to the CRN with the MST tree at $11.2 \%$ and $16.7 \%$ respectively, while outperforming the CRN with the SPT algorithm by $16.7 \%$ and $24.13 \%$ respectively. And finally, at a busy channel $\left(\mathrm{P}_{\mathrm{I}}=0.1\right)$, the PDR performance of CRN with SPT outperformed the CRN with the SMT and MST algorithms by $64.83 \%$ and $131.92 \%$ respectively (Figure 2).

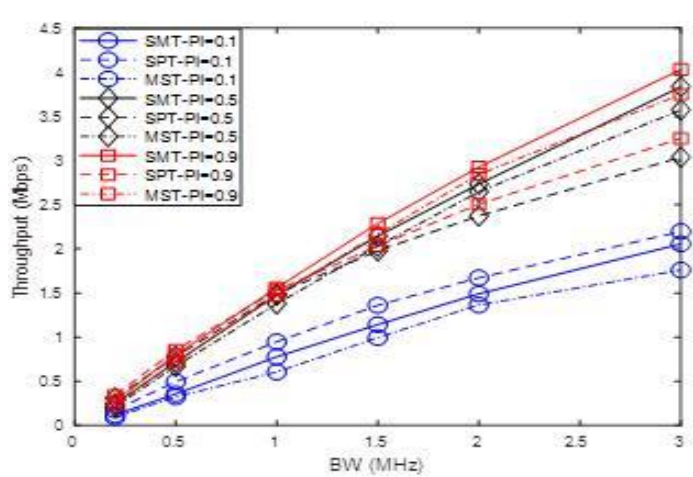

Figure 6. Throughput vs. channel bandwidth

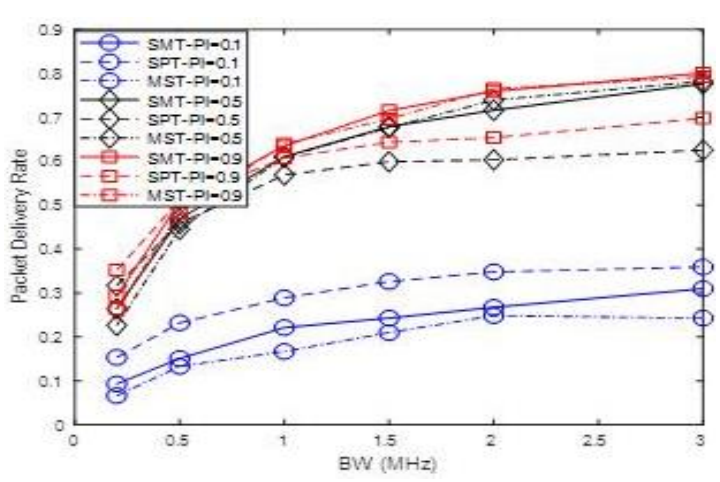

Figure 7. PDR vs. channel bandwidth

\subsection{Performance Evaluation of Multicast MANET CRN under the Impact of Packet Size}

The throughput and PDR performance of multilayer multi hop multicast MANET CRN, with regards to the packet size with three different types of tree algorithms (SMT, SPT and MST), and three values of idle probability $\left[\mathrm{P}_{\mathrm{I}}=0.1,0.5\right.$ and 0.9$]$ are portrayed in Figures 8 and 9 respectively. The channel assignment scheme used for the CRNs is the POS scheme. According to the figures presented, the performance of all CRN tree algorithms, in terms of throughput and PDR, declined as the value of packet size D increased. This decline in performance is due to the fact that at high packet size values, the successful delivery of data from a CR source to destination nodes, calls for an increased channel availability time. This renders the search for the best channel difficult. However, as mentioned in Section (5.1), an increase in the value of $P_{I}$ enhanced the performance of the CRN at the three algorithm protocols. This enhancement came about because the traffic load of PU is low at a high value of $\mathrm{P}_{\mathrm{I}}$. As dispayed in Figure 8, at idle probability $\mathrm{P}_{\mathrm{I}}=0.9$ and 0.5 , the throughput performance of the CRN using the SMT routing tree algorithm, outperformed the CRN with SPT and MST algorithms by $7.81 \%$ and $13.88 \%$ at $\mathrm{P}_{\mathrm{I}}=0.9$, and by $8.84 \%$ and $13 \%$ at $\mathrm{P}_{\mathrm{I}}=0.5$ respectively. However, at $\mathrm{P}_{\mathrm{I}}=0.1$, the CRN with the SPT algorithm outperformed the CRN with the SMT or MST algorithms by $31 \%$ and $46.94 \%$ respectively. As shown in Figure 9, at idle probability $\mathrm{P}_{\mathrm{I}}=0.9$ and 0.5 , the PDR performance of the CNR using the SMT tree routing algorithm is superior to the CRN with SPT and MST algorithms, and is comparable to the MST algorithms at $16.5 \%$ and $8.96 \%$ at $\mathrm{P}_{\mathrm{I}}=0.9$, and by $16.16 \%$ and $6.1 \%$ at $\mathrm{P}_{\mathrm{I}}=0.5$ respectively. However, at $\mathrm{P}_{\mathrm{I}}=0.1$, the CRN with the SPT algorithm outperformed the CRN with the SMT and MST algorithms by $46.17 \%$ and $63.31 \%$ respectively.

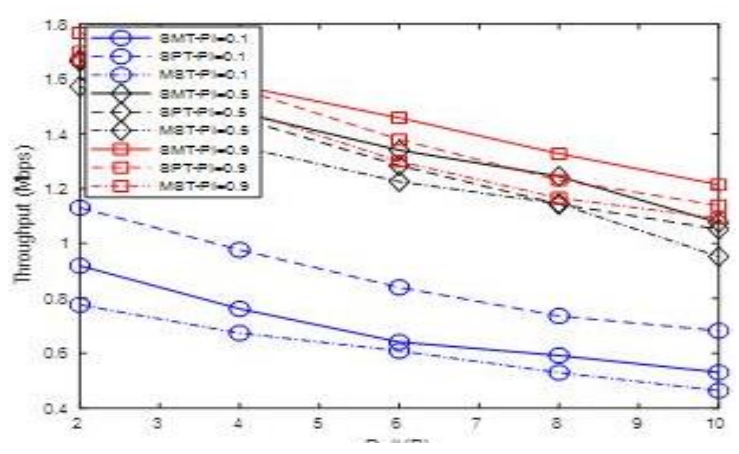

Figure 8. Throughput vs. data packet size

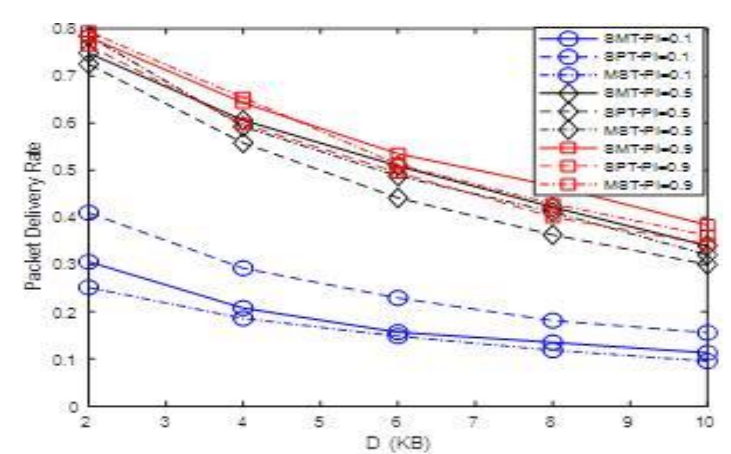

Figure 9. PDR vs. data packet size

Performance comparison of multicast MANET CRN system's based on POS... (Basma Nazar Nadhim) 


\subsection{Performance Evaluation of Multicast MANET CRN under the Impact of Increased Primary Channels}

The throughput and PDR performance of the multilayer multi hop multicast MANET CRN, with regards to the number of primary channels with three different types of tree algorithms (SMT, SPT and MST), and three values of idle probability $\left[\mathrm{P}_{\mathrm{I}}=0.1,0.5\right.$, and 0.9], are presented in Figures 10 and 11 respectively. The channel assignment scheme used for the CRNs is the POS scheme. The figures revealed that the throughput and PDR performances were enhanced by an increase in the number of primary channels. This is because an increase in the number of primary channels creates the opening, for an enhancement in the number of channels available to CR users. As illustrated in Figure 10, for $P_{I}=0.9$ and number of channels $\leq$ 6, the CRN with the SPT algorithm offers a better performance than the CRN with the SMT and MST algorithms. Also, the CRN with the SPT algorithm outperforms the CRN with the SMT and MST algorithms by $85.4 \%$ and $135.7 \%$, and by $36.26 \%$ and $48.36 \%$ at $\mathrm{P}_{\mathrm{I}}=0.1$ and 0.5 respectively. This is attributed to the SPT algorithm using the short path distance between source node and destinations. With the use of the short path distance, the number of hops is minimized to reduce the required transmission time, and increase the value of throughput. As shown in Figure 11, at $\mathrm{P}_{\mathrm{I}}=0.5$ and for number of channels $<6$, in terms of PDR, the SPT algorithm is superior to the SMT and MST algorithms. At $P_{I}=0.9$ the performances of the SMT and MST algorithms are comparable, while outperforming the SPT algorithm. At $\mathrm{P}_{\mathrm{I}}=0.1$, the SPT algorithm outperformed the SMT and MST algorithms by $191.89 \%$ and $328.88 \%$ respectively.

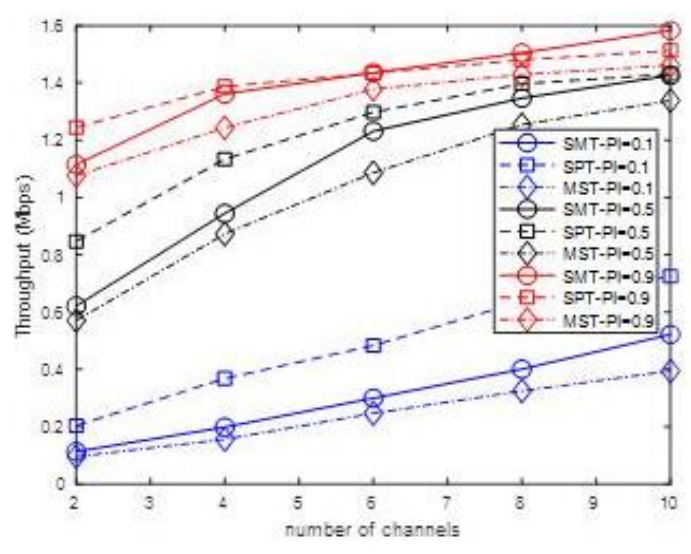

Figure 10. Throughput vs. Number of channels

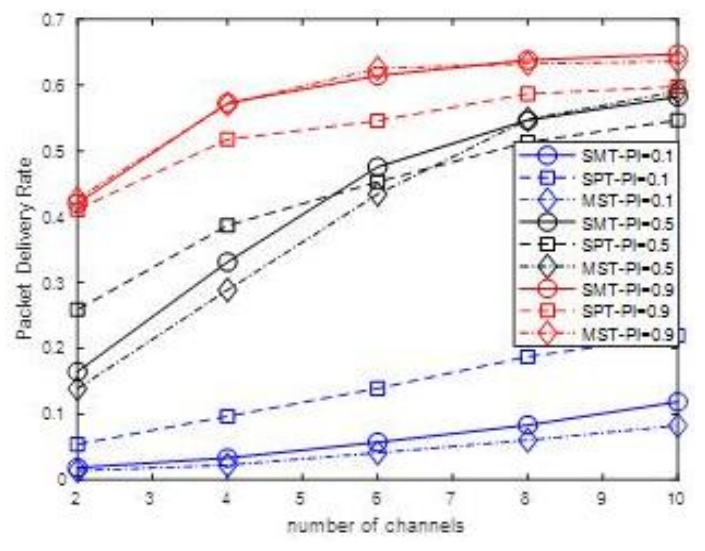

Figure 11. PDR vs. Number of Channels

\subsection{Performance Evaluation of Multicast MANET CRN under the Impact of Increased Transmission Power}

The throughput and PDR performance of multilayer multi hop multicast MANET CRN, with regards to transmission power with three different types of tree algorithms (SMT, SPT and MST), and three values of idle probability $\left[\mathrm{P}_{\mathrm{I}}=0.1,0.5\right.$, and 0.9$]$, are presented in Figures 12 and 13 respectively. POS was used as the channel assignment scheme for all the CNRs. As the transmission power increased, the performance of the CRN for the three algorithms were enhanced, in terms of throughput and PDR. This is attributed to the high value of transmission power reducing the time required for data transmission. As such, more data can be transmitted over each channel. Also, in a circumstance where the traffic load for PU is low, the CRN performance for the three algorithms is enhanced (as mentioned in previous sections). The enhanced throughput gains for the CRN with the SPT algorithm, against the CRN with the SMT and MST algorithms at $\mathrm{P}_{\mathrm{I}}=0.1,0.5$ and 0.9 are $74.82 \%$ and $108.4 \%, 39 \%$ and $61.39 \%$, and $38.93 \%$ and $57.42 \%$ respectively. The improved PDR gains for the SPT algorithm in comparison to the SMT and MST algorithms at $\mathrm{P}_{\mathrm{I}}=0.1$, 0.5 and 0.9 are $78.64 \%$ and $111.38 \%, 11.32 \%$ and $14.24 \%$, and $8.79 \%$ and $13.59 \%$ respectively. 


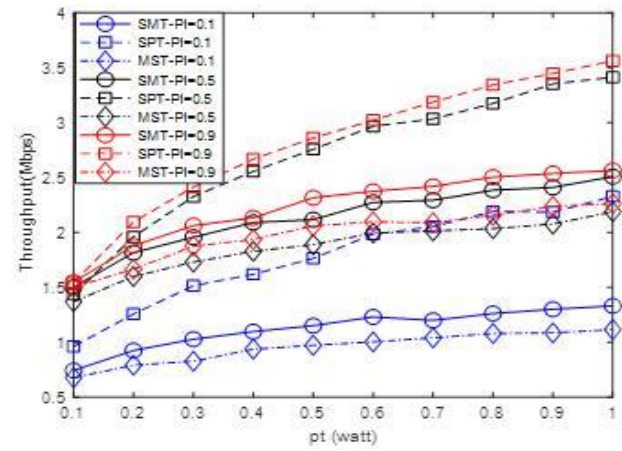

Figure 12. Throughput vs. Transmission Power

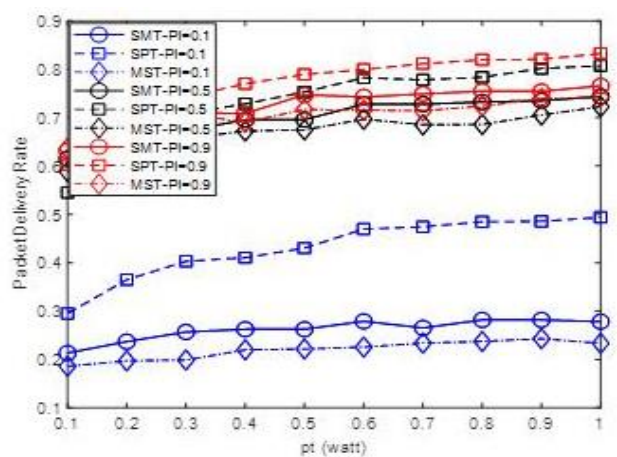

Figure 13. PDR vs. Transmission Power

\subsection{Performance Evaluation of Multicast MANET CRN under the Impact of Path Loss Exponent}

The throughput and PDR performance of multilayer multi hop multicast MANET CRN with regards to path loss exponent, with three different types of tree algorithms (SMT, SPT and MST), and three values of idle probability $\left[\mathrm{P}_{\mathrm{I}}=0.1,0.5\right.$ and 0.9$]$, are presented in Figures 14 and 15 respectively. POS was used as the channel assignment scheme for all the CNRs. As the value of path loss exponent (n) increased, the CRN performance for the three algorithms dipped in terms of throughput and PDR. This dip, which is attributed to the negative influence of the Rayleigh fading channel, greatly reduced the chances of finding the best channel for data transmission. Figures 9 and 10 indicate that at $n>4$, and as the value of $P_{I}$ is increased, the performance of the CRN at the three algorithm protocols was improved, and the performance of the CRN with the SPT algorithm, grew superior to those of the CRN with the SMT or MST algorithm by $172.08 \%$ and $247.81 \%, 114.89 \%$ and $166.3 \%$, and $109.65 \%$ and $160.03 \%$ at idle probability $=0.1,0.5$ and 0.9 respectively in terms of throughput, and by $82.52 \%$ and $117.53 \%, 13.55 \%$ and $20.25 \%$, and $13.17 \%$ and $17.97 \%$ at idle probability $=0.1,0.5$ and 0.9 respectively in terms of PDR. This is attributed to the SPT algorithm's use of the short path distance between the source node, and the destinations. The resulting minimized number of hops reduced the required transmission time, to consequently improve the performance of the CRN with the SPT algorithm.

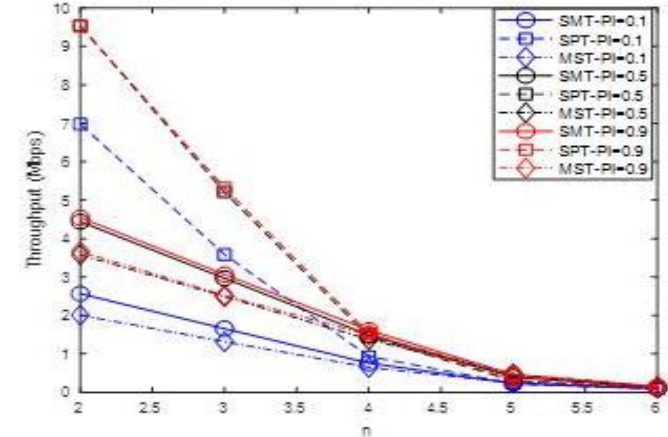

Figure 14. Throughput vs. Path loss exponent

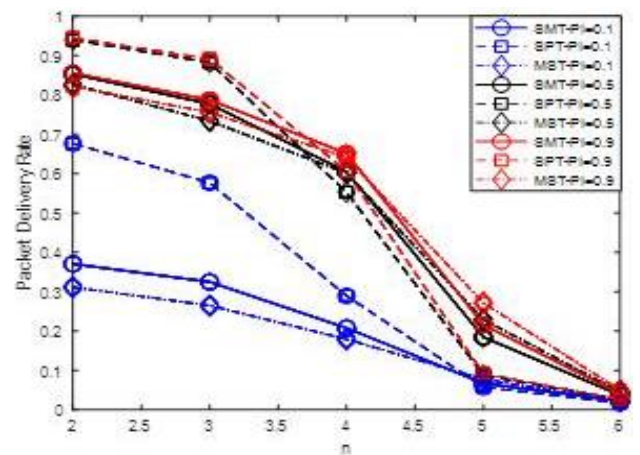

Figure 15. PDR vs. Path loss exponent

\subsection{Performance Evaluation of Multicast MANET CRN under the Impact of Idle Probability}

The throughput and PDR performance of the multilayer multi hop multicast MANET CRN, with regards to the values of idle probability, with three different types of tree algorithms (SMT, SPT and MST), and with POS as the channel assignment scheme in all the CNRs, are presented in Figures 16 and 17 respectively. As indicated in Figure 11, at idle probability< 0.3, the performance of the CRN with the SPT algorithm during a high traffic load of PUs, is superior to the CRNs with the SMT or MST algorithm. As the idle probability increased, the performance of all the CRNs improved. This is attributed to the raised probability, that suitable channels will become available at a low traffic load of PUs. For Idle probability $>$ 0.3, the performance of the SMT algorithm is comparable to those of the SPT and MST algorithms at $4.59 \%$ and $11.4 \%$ respectively. As portrayed in Figure 17, at idle probability $\geq 3$, the CRN with the SPT algorithm, experienced a bigger drop in PDR performance than the CRNs with the SMT or MST algorithm. 


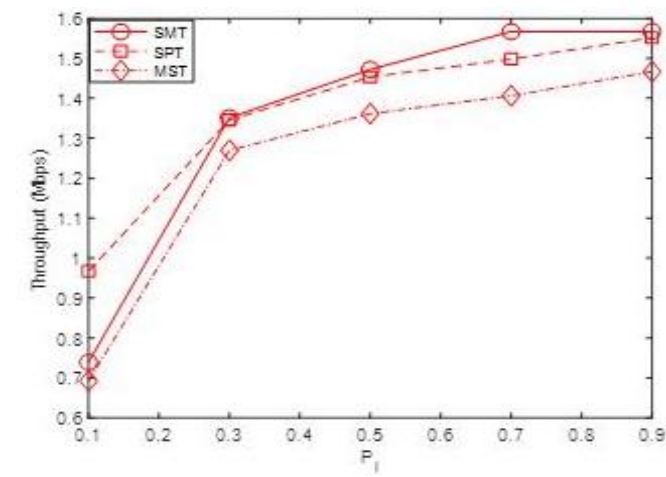

Figure 16. Throughput vs. Idle probability

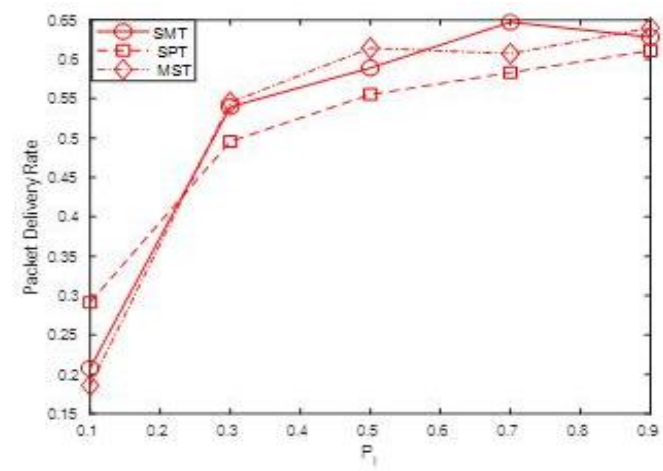

Figure 17. PDR vs. Idle probability

\section{CONCLUSION}

During this investigation, we delved into the use of a multi-layer multi-hop mechanism, with one of three different types of router protocol algorithms (Steiner minimal tree (SMT), shortest path tree (SPT) and minimal spanning tree (MST)), for the construction of multi-cast CRNs. The selection of an efficient channel, for data transmission by CR users, was conducted under the effect of the Rayleigh fading channel. The probability of success (POS) was employed as the channel assignment scheme. Three values for idle probability (i.e. $P_{I}=0.1,0.5$ and 0.9 ), and different network parameters, were used to compare the CRN performance with regard to three routing protocols, in terms of throughput and PDR. According to the simulation results, as BW and D increased, and for $P_{I}=0.5$ and 0.9 , the SMT algorithm delivered a better throughput and PDR performance than the SPT and MST algorithms. At different network parameters, the CRN with the SMT algorithm offered a better performance than the MST algorithm because it used an addition node (Steiner node). The use of the addition note shortened the distance path for the transmission of data from the CR source to the destinations. This enhanced the CRN performance by reducing the time required for transmission. For a high traffic load of PUs $\left(P_{I}=0.1\right)$, and at different network parameters, the CRN with the SPT algorithm displayed a better performance than the CRN with the SMT or MST algorithm. This applied for both throughput and PDR performances. It should be noted that as the value of $\mathrm{P}_{\mathrm{I}}$ is increased; the performance of the $\mathrm{CRN}$ at the three algorithm protocols is enhanced in terms of throughput and PDR. This is because at a high value of $\mathrm{P}_{\mathrm{I}}$, the probability that suitable channels (with a low traffic load of PU) will be available for transmission by CR users, is raised.

\section{ACKNOWLEDGEMENTS}

The authors would like to thank Mustansiriyah University (www.uomustansiriyah.edu.iq) BaghdadIraq for its support in the present work.

\section{REFERENCES}

[1] Y.C. Liang, Y. Zeng, E. C.Y. Peh and A. T. Hoang, “ Sensing-Throughput Tradeoff for Cognitive Radio Networks”, IEEE Transactions On Wireless Communications, vol. 7, no. 4, pp. 1326- 1337,APRIL 2008.

[2] M. K. Kaushik, Y. Yoganandam, S. K. Sahoo, " Sensing and Sharing Schemes for Spectral Efficiency of Cognitive Radios", International Journal of Electrical and Computer Engineering (IJECE), vol. 8, no. 5, pp. 2934-2941, October 2018.

[3] Sh. D. Borde, K. R. Joshi, "Enhanced signal detection algorithm using trained neural network for cognitive radio receiver ", International Journal of Electrical and Computer Engineering (IJECE),vol. 9, no. 1,pp. 323-331, February 2019.

[4] I. F. Akyildiz, W.Y. Lee, M. C. Vuran and Shantidev Mohanty, "NeXt generation/dynamic spectrum access/cognitive radio wireless networks: A survey", Computer Networks, vol. 50, no. 13, pp. 2127-2159, May 2006.

[5] V. Balajia, P. Kabra, P. V. P. K. Saieesh, C. Hota and G. Raghurama, "Cooperative Spectrum Sensing in Cognitive Radios using Perceptron Learning for IEEE 802.22 WRAN", Eleventh International Multi-Conference on Information Processing-2015 (IMCIP-2015), Procedia Computer Science, vol. 54, pp. 14 - 23,2015.

[6] H. Al-Mahdi and Y. Fouad, "Design and analysis of routing protocol for cognitive radio ad hoc networks in heterogeneous environment", International Journal of Electrical and Computer Engineering (IJECE), vol. 9, no. 1, pp. 341-351, February 2019. 
[7] Y.C. Liang, K.C. Chen, G. Y. Li and P. Mähönen, “ Cognitive Radio Networking and Communications: An Overview”, IEEE Transactions On Vehicular Technology, vol. 60, no. 7, pp. 3386-3407, September 2011.

[8] P. Thakur, A. Kumar, S. Pandit, G. Singh and S.N. Satashia, "Performance analysis of cognitive radio networks using channel-prediction-probabilities and improved frame structure", Digital Communications and Networks, vol.4, pp. 287-295,2018.

[9] J. Ma, G. Zhao, and Ye (Geoffrey) Li, "Soft Combination and Detection for Cooperative Spectrum Sensing in Cognitive Radio Networks" IEEE Transactions On Wireless Communications, vol. 7, no. 11, pp. 4502-4507, November 2008.

[10] Z. Quan, S. Cui and A. H. Sayed, "Optimal Linear Cooperation for Spectrum Sensing in Cognitive Radio Networks", IEEE Journal of Selected Topics in Signal Process., vol. 2, no. 1, pp.28-40, February 2008.

[11] Y.u, L. X. Cai and X. (Sherman) Shen, "Spectrum-Aware Opportunistic Routing in Multi-Hop Cognitive Radio Networks", IEEE Journal On Selected Areas In Communications, vol. 30, no. 10, pp. 1958-1968, November 2012.

[12] J. J. Dhivya, M. Ramaswami, "Ingenious Method for Conducive Handoff Appliance in Cognitive Radio Networks", International Journal of Electrical and Computer Engineering (IJECE), vol. 8, no. 6, pp. 5195-5202, December 2018

[13] S. Lakhal and Z. Guennoun, "Equity-based free channels assignment for secondary users in a cognitive radio network", International Journal of Electrical and Computer Engineering (IJECE), vol. 9, no. 3, pp. 2057-2063, June 2019

[14] R. A. H. Suliman, K. H. Bilal and I. Elemam, "Review Paper on Cognitive Radio Networks", Journal of Electrical \& Electronic Systems, vol.7,issue1,pp.1-3, 2018.

[15] A. S. Khobragade and R. D. Raut, "Hybrid Spectrum Sensing Method for Cognitive Radio", International Journal of Electrical and Computer Engineering (IJECE),vol. 7, no. 5, pp. 2683-2695, October 2017.

[16] C. Gao, Y. Shi,Y. T. Hou,H. D. Sherali, and H. Zhou,"Multicast Communications in Multi-Hop Cognitive Radio Networks", IEEE Journal On Selected Areas In Communications, vol. 29, no. 4, pp. 784-793, APRIL 2011.

[17] L. Junhai, X. Liu and Y. Danxia, "Research on multicast routing protocols for mobile ad-hoc networks", Computer Networks, vol.52, pp.988-997,2008.

[18] P. Gaur, "Implementation of Multicast Routing Using Genetic Algorithm", IJCSMC, vol. 2, issue. 5, pp.226 - 231, May 2013.

[19] G. prasad, Vimala, G. patil and Margesh keskar, "Improving performance using Ternary Tree Multicast Routing Protocol for MANET”, IRJET, vol. 04, issue 10, pp. 1596-1601, Oct -2017

[20] M. Nagaratna, Dr. V. Kamakshi Prasad and Dr. C. Raghavendra Rao, "Performance Evaluation of Tree - Based Multicast Routing Protocols in MANETs", IJCST, vol. 2, issue 3,pp. 558-562 September 2011.

[21] S. Sumathy, B. Yuvaraj and E S. Harsha, "Analysis of Multicast Routing Protocols: Puma and Odmrp", IJMER, vol.2, issue.6, pp-4613-4621, Nov-Dec. 2012.

[22] O. S. Badarneh, H. B. Salameh, "Probabilistic quality-aware routing in cognitive radio networks under dynamically varying spectrum opportunities", Computers and Electrical Engineering vol.38,pp. 1731-1744,2012.

[23] J. Qadir, A. Baig, A. Ali, and Q.Shafi, "Multicasting in cognitive radio networks: Algorithms, techniques and protocols", Journal of Network and Computer Applications, vol.45,pp.44-61,2014.

[24] R. Novak, J. Rugelj and G. Kandus., "Steiner tree based distributed multicast routing in networks", In X. Cheng and D.-Z. Du, editors, Steiner Trees in Industry, vol. 11 of Combinatorial Optimization, pp. 327-351. Kluwer Academic Publishers, Dordrecht, 2001.

[25] M. Wadhwa and Dr.K. Saxena, "Steiner Tree: approach applying for shortest path in selected network", IOSR Journal of Computer Engineering, vol. 15, issue 6,pp.23-26, Nov. - Dec. 2013.

[26] H. Khalife, S. Ahuja, N. Malouch and M. Krunz, "Probabilistic Path Selection in Opportunistic Cognitive Radio Networks", in Proceedings of IEEE Global Telecommunications Conference (GLOBECOM), pp. 1-5, Nov 2008.

[27] W. Kim, S. Y. Oh, Mario Gerla and Joon-Sang Park, "Cocast: Multicast Mobile Ad Hoc Networks Using Cognitive Radio", IEEE Military Communications Conferences (MILCOM 2009), Boston, MA,pp.1-7, October 2009.

[28] W. Ren, X. Xiao and Q. Zhao, "Minimum-Energy Multicast Tree in Cognitive Radio Networks", Conference Record of the Forty-Third Asilomar Conference on Signals, Systems and Computers, pp. 312-316, Pacific Grove, CA, 1-4 Nov. 2009.

[29] D. Hu, S. Mao, and J. H. Reed, "On Video Multicast in Cognitive Radio Networks", INFOCOM, IEEE, pp. 2222-2230, Rio de Janeiro, 19-25 April 2009.

[30] H. B. Salameh, "Rate-maximization Channel Assignment Scheme for Cognitive Radio Networks", in IEEE Global Telecommunications Conference (GLOBECOM 2010), pp. 1-5, Dec 2010.

[31] H. M. Almasaeid, T. H. Jawadwala, and A. E. Kamal, "On-Demand Multicast Routing in Cognitive Radio Mesh Networks", Global Telecommunications Conference, GlobeCom, pp. 1-5, Miami, FL, 6-10 Dec. 2010.

[32] L. T. Tan and L. B. Le, "Channel Assignment for Throughput Maximization in Cognitive Radio Networks", IEEE Wireless Communications and Networking Conf.: MAC and Cross-Layer Design, 2012, pp. 1427-1431.

[33] T. Le, V. Rabsatt and Mario Gerla, "Cognitive Routing with the ETX Metric", Ad Hoc Networking Workshop (MED-HOC-NET), 2014 13th Annual Mediterranean,pp.188 - 194, June 2014.

[34] Y. Mhaidat, M. Alsmirat, O. S. Badarneh, Y. Jararweh and H. A. B. Salameh, "A Cross-Layer Video Multicasting Routing Protocol for Cognitive Radio Networks", seventh international workshop on selected topics in mobile and wireless comuting,pp.384-389, 2014.

[35] M. M. Ali and H. B. Salameh., "Multi-layer Mechanism for Multicast Routing in Multi-hop Cognitive Radio Network", M.S. thesis, Dept. Telecommunication Eng., Yarmouk University Hijjawi Faculty for Engineering Technology, Jordan, Julay 2015. 
[36] M. Al-rubaye, H. B. Salameh., Y. Jararweh "Minimum spanning tree - based multicast routing protocol for dynamic spectrum access networks: A multi-layer probabilistic approach", in Proc. 7th International Conf. on Computer Science and Information Technology (CSIT), 2016, pp.1-6.

[37] R. Z.Abu Samra and H. B. Salameh, "A Dynamic Multi-Cast Routing Algorithm for Opportunistic Networks: Implementing The Expected Transmission Count Metric", M.S. thesis, Dept. Telecommunication Eng., Yarmouk University Hijjawi Faculty for Engineering Technology, Jordan, May 2016.

[38] B. Ye Wu, K.M. Chao., Spanning Trees and Optimization Problems, Library of Congress Cataloging in Publication Data, Chapman and Hall/CRC,2004, ch.7, pp.147-150.

[39] V.K.Balakrishnan, Schaum's outline of Theory and Problems of Graph Theory, The McGraw-Hill Companies,1997,ch5.,pp.121-122. 